\title{
Effectiveness of Inactivated COVID-19 Vaccines Against Symptomatic, Pneumonia, and Severe Disease Caused by the Delta Variant: Real World Study and Evidence - China, 2021
}

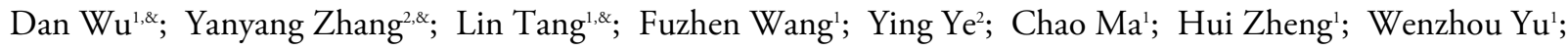 \\ Lei Cao'; Yifan Song'; Abuduwaili Reyimu ${ }^{3,4}$; Xiaoxiao Zhang²; Haifeng Wang'; Yifei $\mathrm{Nie}^{2}$; Mingxia $\mathrm{Lu}^{2}$; \\ Muge $\mathrm{Qi}^{2}$; Jun $\mathrm{Li}^{2}$; Ruolin Wang²; Kaichao Yang²; Changshuang Wang'; Lawrence Everett Rodewald'; \\ Geroge Fu Gao'; Zhijie An ${ }^{1, *}$; Zundong Yin ${ }^{1, *}$
}

\section{Summary}

What is already known about this topic?

Effectiveness of China's 2 inactivated vaccines (BBIBP-

$\mathrm{CorV}$ and CoronaVac) against pre-Delta severe acute respiratory syndrome coronavirus-2 (SARS-CoV-2) variants ranged from $47 \%$ to over $90 \%$, depending on the clinical endpoint, and with greater effectiveness against more severe coronavirus disease 2019 (COVID-19). During an outbreak in Guangdong, inactivated vaccine effectiveness (VE) against the Delta variant was $70 \%$ for symptomatic infection and $100 \%$ for severe COVID-19. However, separate or combined VE estimates for the two inactivated vaccines against Delta are not available.

What is added by this report?

In an outbreak that started in a hospital, VEs of completed primary vaccination with inactivated COVID-19 vaccines against symptomatic COVID-19, COVID-19 pneumonia, and severe COVID-19 caused by the Delta variant were $51 \%, 61 \%$, and $82 \%$. Completed primary vaccination reduced the risk of progressing from mild to moderate or severe COVID-19 by $74 \%$. VE estimates for BBIBP-CorV and CoronaVac or combined vaccination were similar, and partial vaccination was ineffective.

What are the implications for public health practice?

Completed primary vaccination with either of the 2 inactivated COVID-19 vaccines reduces risk of symptomatic COVID-19, COVID-19 pneumonia, and severe COVID-19 caused by the Delta variant. Completion of the completed primary vaccination with two doses is necessary for protection from Delta.

\section{INTRODUCTION}

Knowledge of real-world performance of coronavirus disease 2019 (COVID-19) vaccines is critically important for informing pandemic vaccination strategy and policy. As severe acute respiratory syndrome coronavirus-2 (SARS-CoV-2) evolves and variants emerge that vary in severity of illness, transmissibility, and immune escape potential, every opportunity must be taken to measure vaccine effectiveness (VE) in realworld studies so that policy can be adjusted to keep upto-date with variants, duration of vaccine-induced protection, and implementation of booster doses. Currently in China, outbreaks offer the only opportunities to measure VE. At the end of July and August 2021 there was a 167-case, hospital-centered, Delta-variant outbreak in Henan Province. We conducted a retrospective cohort study among 1,462 close contacts of SARS-CoV-2-infected individuals in the outbreak who were quarantined and systematically tested for infection over a 2-week interval. Vaccination status was verified by the national vaccine information system. We found that completed primary series vaccination $\mathrm{VE}$ with 2 doses of inactivated vaccines was $51 \%$ against symptomatic infection, $61 \%$ against COVID-19 pneumonia, and $82 \%$ against severe COVID-19. Completed primary vaccination reduced the risk of progressing from mild to moderate or severe COVID-19 by $74 \%$. VE levels were similar for BBIBP-CorV and CoronaVac vaccines. We concluded that both inactivated vaccines retained effectiveness against the Delta variant, consistent with efficacy clinical trials, and that these vaccines can continue to be used to protect individuals and prevent or control disease during the pandemic.

COVID-19 vaccines are important tools for COVID-19 pandemic management. Five vaccine development techniques are being used to develop and produce COVID-19 vaccines in China: whole-virus 
inactivated, adenovirus vectored, recombinant protein subunit, nucleic acid, and attenuated influenza virus vectored vaccines. Inactivated vaccines are in the most widespread use in China (over 85\% all COVID-19 vaccine doses administered), with two manufacturers - Sinopharm (BBIBP-CorV) and Sinovac (CoronaVac) - providing the largest share. These two vaccines have been listed by the World Health Organization (WHO) for emergency use and are in widespread use globally.

China initiated a large domestic COVID-19 vaccination campaign on December 15, 2020. In June and July 2021, the Delta (B.1.617.2) variant began to cause outbreaks in China. BBIBP-CorV and CoronaVac vaccines are frequently used in the same population at the same time during the campaign, providing opportunity for single-study estimates of VE against the Delta variant by vaccine brand. Taking advantage of a Delta variant outbreak in Zhengzhou city of Henan Province, we evaluated VE against symptomatic COVID-19, COVID-19 pneumonia, and severe COVID-19 by time since vaccination and vaccine brand.

\section{METHODS}

We evaluated VE using a retrospective cohort study among close contacts of individuals who were polymerase chain reaction (PCR)-confirmed to have been infected with SARS-CoV-2 in an outbreak that started in a hospital in Zhengzhou and spread to the community. We estimated the effectiveness of vaccination to prevent progression of illness by comparing the odds of vaccination of asymptomatic and mild cases versus the odds of vaccination in moderate and severe cases using an age-stratified analysis.

\section{Setting and Subjects}

The study setting was Zhengzhou, the capital city of Henan Province. As of July 17, 2021, 74.8\% of Zhengzhou's 18-59-year-old residents were completed vaccinated and $46.4 \%$ of residents $\geq 60$ years old were completed vaccinated. Over $98 \%$ of all adults who were vaccinated received an inactivated COVID-19 vaccine. We drew our subjects from close contacts of people with laboratory-confirmed SARS-CoV-2 infection.

For VE evaluation, we included only close contacts, 18 years and older, who had documented contact or exposure opportunities (contact with one or more confirmed cases or asymptomatic infections in the same public space, without protection, within close distance, within up to five days before illness onset for symptomatic cases or were identified by the first positive specimen for asymptomatic cases), and no history of COVID-19 infection. We excluded individuals vaccinated with vaccines other than BBIBP-CorV or CoronaVac. For analysis of impact of vaccination on risk of severe COVID-19, we included all infected individuals who were 18 years of age and older.

\section{Vaccination Status}

We considered vaccinations to be valid only if they were documented in the national or the provincial Immunization Information System. Subjects were categorized into an unvaccinated group, a partiallycompleted vaccinated group, and a completed primary vaccinated group based in part on compliance with technical guidelines from China CDC's COVID-19 Vaccines Technical Working Group (1). The unvaccinated group consisted of individuals who did not receive any COVID-19 vaccines before their last known contact with a confirmed case. The partiallycompleted vaccination group consisted of individuals who had received either 1 dose of a COVID-19 inactivated vaccine or had received 2 doses of inactivated vaccines with receipt of the second dose less than 14 days before exposure to an infected individual. The completed primary vaccination group consisted of individuals who completed 2 doses of inactivated vaccine 14 days or more before exposure to an infected individual.

\section{Outcomes}

We evaluated 3 outcomes: symptomatic COVID19, COVID-19 pneumonia, and severe COVID-19. Case classifications were based on the COVID-19 Prevention and Control Protocol (eighth edition) (2), and COVID-19 Diagnosis and Treatment Protocol (Trial eighth edition) (3): asymptomatic, mild, moderate, severe, and critically severe. Symptomatic illness included mild, moderate, severe, and criticallysevere cases. COVID-19 pneumonia included moderate, severe, and critically severe cases with evidence of pneumonia. Severe COVID-19 included severe and critically-severe cases. 


\section{Statistical Analyses}

To calculate unadjusted VE, the relative risk (RR) of each outcome was calculated in reference to the unvaccinated group; VE was 1-RR. Gender, age grouping (18-59 years old and $\geq 60$ years old), and presence of underlying disease(s) were considered potentially confounding variables in multivariate analyses. We used a generalized linear model with a binomial distribution and log link function to calculate adjusted risk ratios (aRR) which were used to calculate adjusted VE (aVE).

To estimate the effectiveness of vaccination for preventing severe COVID-19, we determined the odds of vaccination for subjects with asymptomatic or mild infections (combined) and for subjects with moderate or worse severity infections (combined); effectiveness to prevent severe infection was one minus the ratio of these odds. All data analyses were performed with SAS software (version 9.4, SAS Institute Inc., Cary, NC, USA).

\section{Ethical Review}

COVID-19 is considered a Level 2 infectious disease and is managed as a Level 1 infectious disease. Investigations into outbreaks of COVID-19, including estimating effectiveness of vaccination, are considered public health responsibilities and are exempt from ethical committee review.

\section{RESULTS}

\section{Outbreak, Cases, and Subjects}

On July 31, 2021, a local cluster of COVID-19 cases was identified through PCR screening in a hospital. The virus was isolated and sequenced, showing that the cluster was caused by the Delta variant. The epidemic was managed in accordance with the Protocol for Prevention and Control of COVID19; the last case occurred on August 24. The epidemic consisted of 167 infections, 166 were symptomatic and 1 was asymptomatic; 139 cases were in Zhengzhou. A total of 14 infections were among children below 18 years of age, and 8 were among individuals vaccinated with a vaccine other than BBIBP-CorV or CoronaVac.

For VE evaluation, 1,462 close-contacts were identified including 107 cases that were ultimately diagnosed with PCR-confirmed SARS-CoV-2 infection; 473 close contacts were unvaccinated, and 288,455 , and 246 were vaccinated with BBIBP-CorV, CoronaVac, or both vaccines (one dose of each, in either order), respectively. Table 1 shows the breakdown of the close contacts by vaccination status (unvaccinated, full, or partial vaccination), vaccine brand, age group, presence of comorbidities, and infection status with clinical outcome. Among the close contacts, 42 were HIV-positive (10 individuals) or had tuberculosis (32 individuals); 33 of these 42 close contacts had not been vaccinated, 5 had been partially vaccinated, and 4 completed 2-dose vaccination series; 35 developed infection (11 mild, 17 moderate, 6 severe, and 1 critically severe).

\section{Vaccine Effectiveness and Severity}

Table 2 shows univariate (unadjusted) analyses of VE by vaccination status, age group, and brand of vaccine against three clinical outcomes - symptomatic COVID-19, COVID-19 pneumonia, and severe COVID-19. For adjusted vaccine effectiveness, multivariable regression analyses controlled for gender, presence of underlying conditions (comorbidities), and age group (18-59 and 60+ years).

Table 3 shows adjusted and unadjusted VE by vaccine brand and vaccination status against the 3 clinical outcomes. Completed primary vaccination VEs for adults 18 years and over were $50.54 \%$ against symptomatic COVID-19, 61.4\% against COVID-19 pneumonia, and $82.41 \%$ against severe COVID-19. Partial vaccination had non-statistically-significant VEs against the clinical outcomes.

Table 4 shows adjusted and unadjusted VE by time between vaccination and exposure to SARS-CoV-2. Of the 784 people who completed primary vaccination, the average time from completing full vaccination (plus 14 days) to becoming a close contact was 76 days (range, 14-193 days); 572 (72.96\%) completed primary vaccination three months or less before becoming a close contact, and 212 people $(27.04 \%)$ had intervals 4 to 6 months following full vaccination. The adjusted VE of full vaccination against symptomatic COVID-19 was $52.32 \%$ for $\leq 3$-month intervals and $49.95 \%$ for $4-6$-month intervals (both statistically significant); against COVID-19 pneumonia, VEs were $67.08 \%$ for 4-6-month intervals; and against severe COVID-19, VEs were $80.64 \%$ for $\leq 3$-month intervals. There were no severe cases for longer intervals.

Table 5 shows severity of COVID-19 by vaccination status. The 145 infected individuals aged $\geq 18$ years were categorized as having mild (asymptomatic and mild cases together) or pneumonia/severe (moderate, severe, and critically severe combined together) 


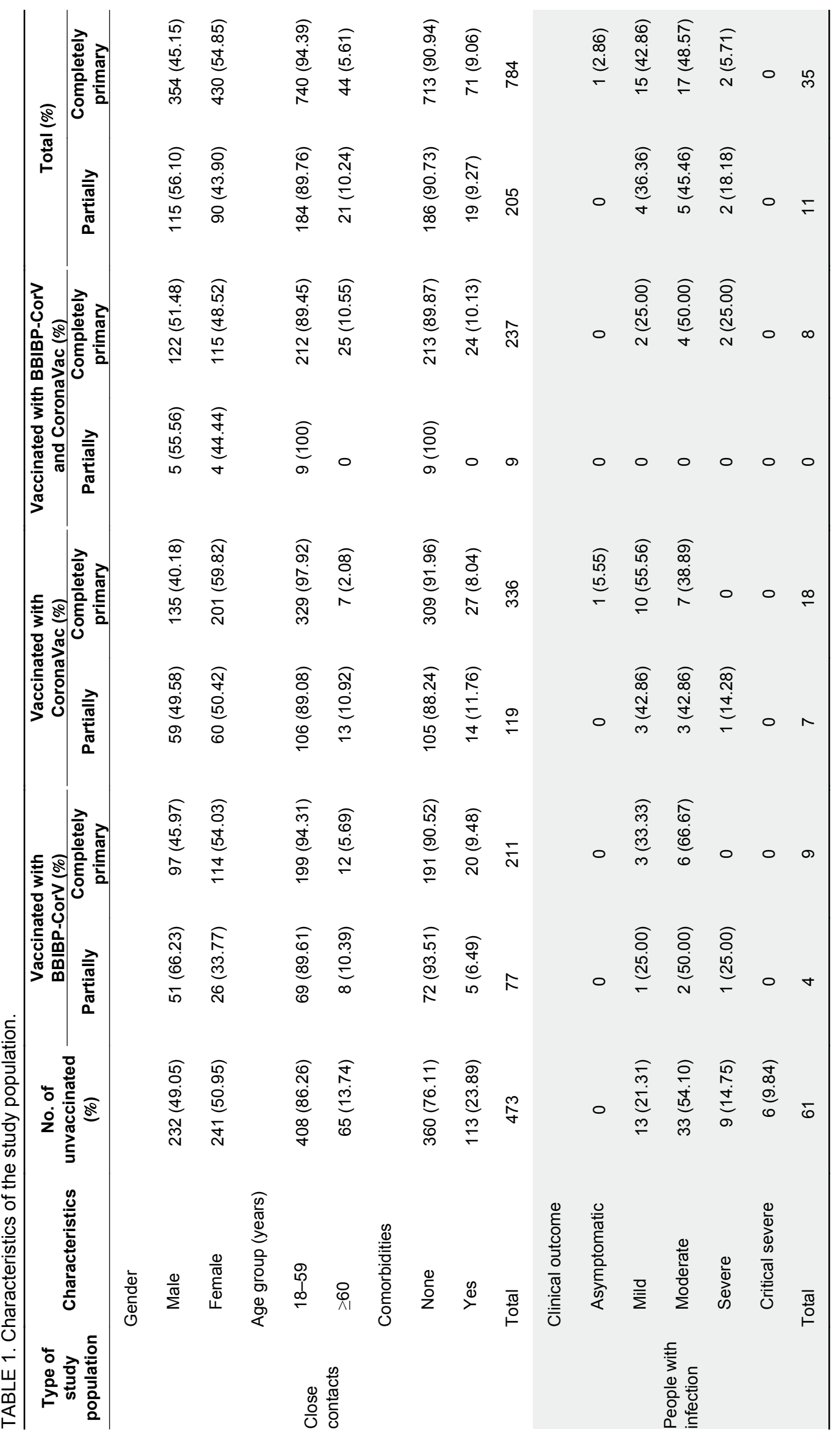




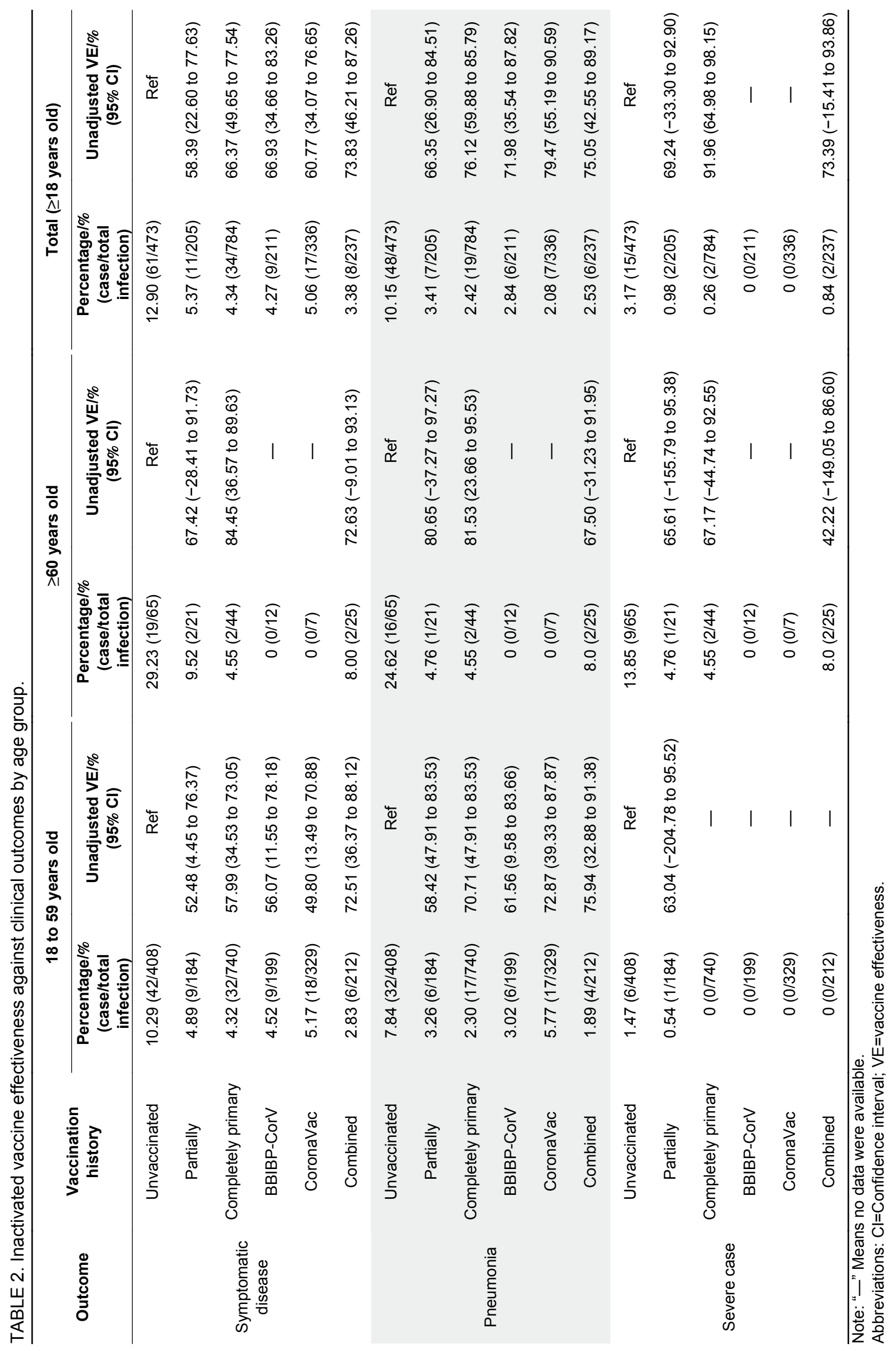




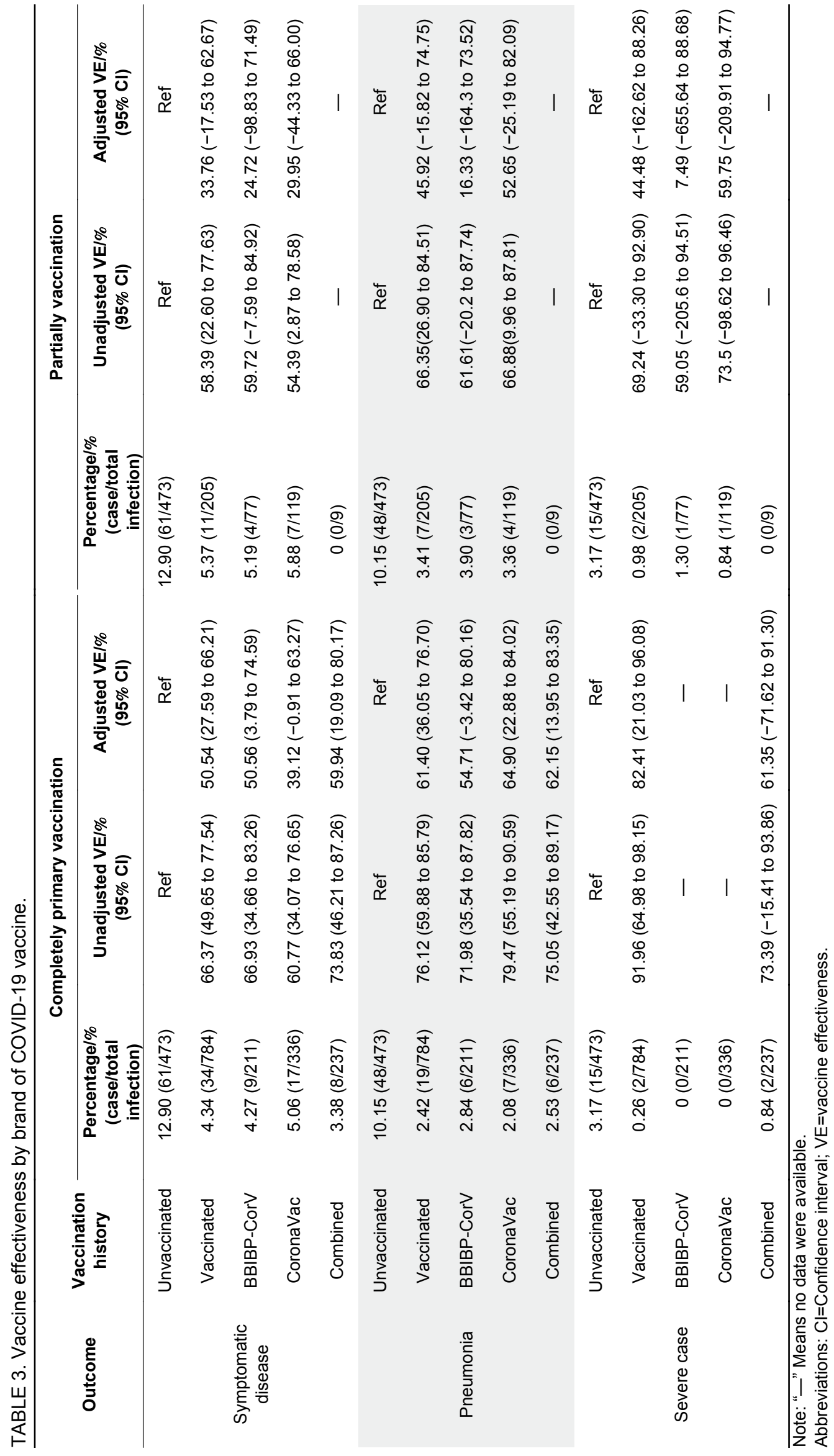




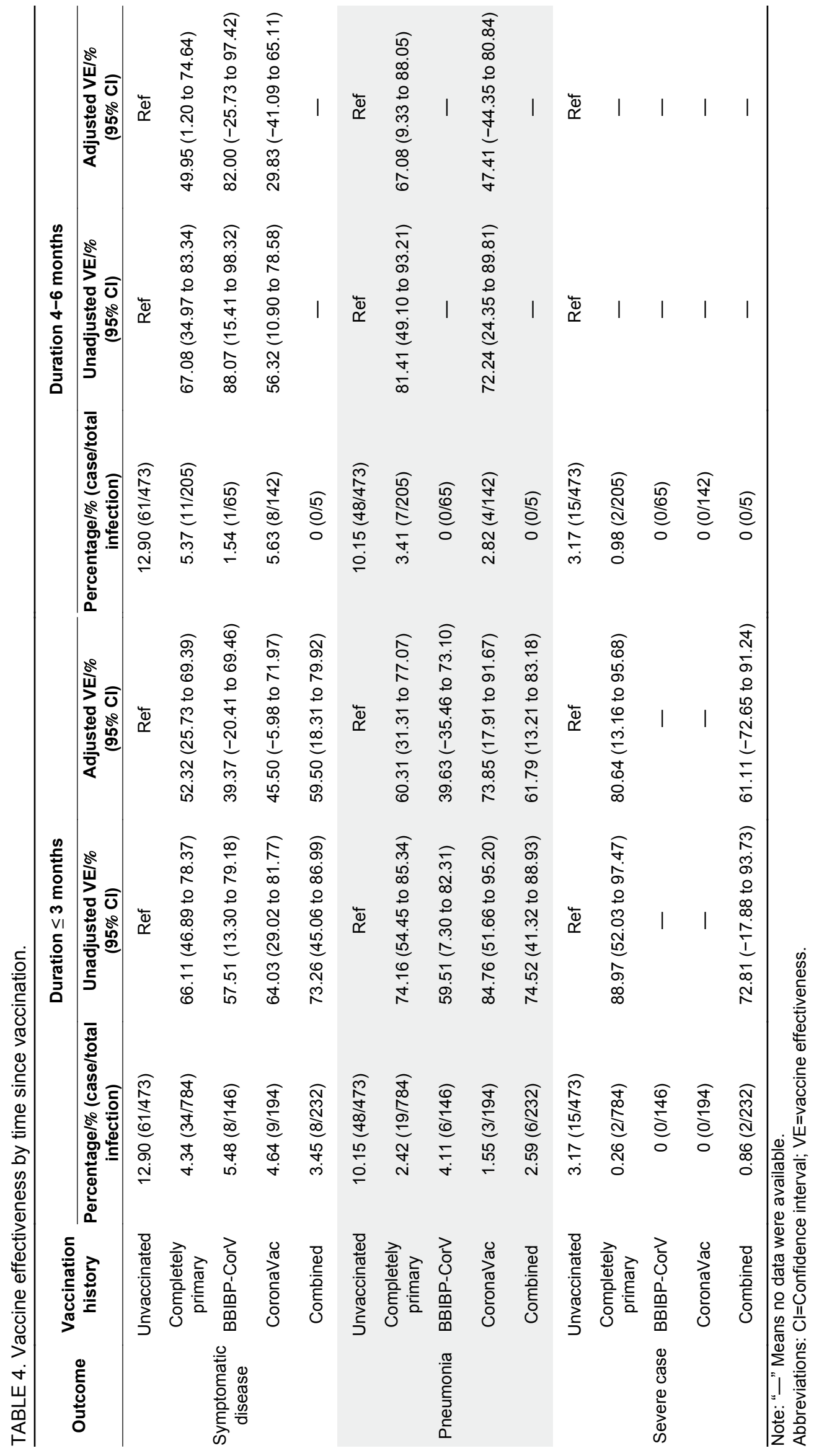


TABLE 5. Severity of illness by vaccination status.

\begin{tabular}{|c|c|c|c|c|c|c|c|}
\hline \multirow[t]{2}{*}{ Age groups } & \multirow{2}{*}{$\begin{array}{l}\text { Vaccination } \\
\text { history }\end{array}$} & \multicolumn{2}{|c|}{$\begin{array}{c}\text { Mild cases } \\
\text { (asymptomatic+mild) } \\
\end{array}$} & \multicolumn{2}{|c|}{$\begin{array}{c}\text { Pneumonia } \\
\text { (moderate+severe) }\end{array}$} & \multirow[t]{2}{*}{ OR } & \multirow[t]{2}{*}{$95 \% \mathrm{Cl}$} \\
\hline & & $\mathbf{n}$ & $\%$ & $\mathbf{n}$ & $\%$ & & \\
\hline \multirow{4}{*}{$\begin{array}{c}18-59 \\
\text { years old }\end{array}$} & Unvaccinated & 13 & 30.95 & 40 & 55.56 & Ref & Ref \\
\hline & Partially & 6 & 14.29 & 10 & 13.89 & 0.54 & 0.16 to 1.78 \\
\hline & Completely primary & 23 & 54.76 & 22 & 30.56 & 0.31 & 0.13 to 0.73 \\
\hline & Sub-total & 42 & 100 & 72 & 100 & & \\
\hline \multirow{4}{*}{$\begin{array}{l}\geq 60 \\
\text { years old }\end{array}$} & Unvaccinated & 3 & 50.00 & 21 & 84.00 & Ref & Ref \\
\hline & Partially & 1 & 16.67 & 1 & 4.00 & 0.16 & 0.002 to 14.92 \\
\hline & Completely primary & 2 & 33.33 & 3 & 12.00 & 0.23 & 0.017 to 3.81 \\
\hline & Sub-total & 6 & 100 & 25 & 100 & & \\
\hline \multirow{4}{*}{ Total } & Unvaccinated & 16 & 33.33 & 61 & 62.89 & Ref & Ref \\
\hline & Partially & 7 & 14.58 & 11 & 11.34 & 0.41 & 0.14 to 1.23 \\
\hline & Completely primary & 25 & 52.08 & 25 & 25.77 & 0.26 & 0.12 to 0.57 \\
\hline & Sub-total & 48 & 100 & 97 & 100 & & \\
\hline
\end{tabular}

Abbreviations: $\mathrm{OR}=$ odds ratio; $\mathrm{Cl}=$ confidential interval.

COVID-19. Compared with the unvaccinated, completed primary vaccination reduced the risk of pneumonia/severe COVID-19 by 74\% [95\% confidence interval (CI): $43 \%$ to $88 \%$ ]. By age grouping, pneumonia/severe risk was reduced by $69 \%$ (95\% CI: $27 \%$ to $87 \%$ ) among $18-59$-year-olds and by $77 \%$ (95\% CI: $-281 \%$ to $98 \%$, not statistically significant) among people over 60 years old.

\section{DISCUSSION}

To our knowledge, this is the first single-study evaluation of brand-specific vaccine effectiveness of the two China-produced inactivated COVID-19 vaccines approved for emergency use by WHO. Our study showed that completed primary vaccination with BBIBP-CorV and CoronaVac inactivated vaccines separately or combined had similar effectiveness against the Delta variant as was seen in the Phase 3 placebocontrolled licensure clinical trials that were conducted when the ancestral SARS-CoV-2 strain circulated (4-5). In this outbreak, centered in a hospital, inactivated VE levels against symptomatic COVID-19, COVID-19 pneumonia, and severe COVID-19 caused by the Delta variant were $51 \%, 61 \%$, and $82 \%$. Completed primary vaccination reduced the risk of progressing from mild to moderate or severe COVID19 by $74 \%$.

$\mathrm{VE}$ of the inactivated vaccine against symptomatic COVID-19 caused by the Delta variant in this outbreak was similar to VE estimates in other real- world studies against ancestral or earlier variants, also shown in other types of vaccines. A cohort study in Peru showed BBIBP-CorV VE against infection and death from pre-Delta variants of concern were $50 \%$ and $94 \%$, respectively (6). A cohort study in Hungary estimated BBIBP-CorV effectiveness to be $87 \%$ against symptomatic COVID-19 and 88\% against COVID-19 death caused by pre-Delta variants. A cohort study in Chile investigated the VE of CoronaVac found that the adjusted VE of completely primary vaccination was $65.9 \%$ against COVID-19, $87.4 \%$ against hospitalization, $90.3 \%$ against ICU admission, and $86.3 \%$ against COVID-19-related death (7). Inactivated vaccine effectiveness against COVID-19 pneumonia was similar to what was observed in a Delta-variant outbreak in Guangdong Province $(61.4 \%$ vs. $69.5 \%)$ (8). Other COVID-19 vaccines, including mRNA and adenovirus-vectored retain effectiveness against the Delta variant (9).

VEs for BBIBP-CorV and CoronaVac or combined vaccination were similar, and partial vaccination was not effective. We used a case-control design to determine the ability of inactivated vaccines to prevent progression from mild to moderate or severe COVID-19, finding that complete primary vaccination reduced the risk of progression by $74 \%$, thus demonstrating good effectiveness in this real world study, although there were differences for the point estimates of VE against symptomatic disease and against pneumonia.

We used a case-control design to determine the 
ability of inactivated vaccines to prevent progression from mild to moderate or severe illness, finding that complete primary vaccination reduced the risk of progression by $74 \%$, thus demonstrating good effectiveness in this real world study.

Our study has program and policy implications. First, evidence of effectiveness of both inactivated vaccines against the Delta variant, when given separately or together, supports continuation of the vaccination campaign to ensure that entire target populations are reached with full-series vaccination. Although completed primary series in the same brand are preferred, our study shows that interchangeable schedules are also effective. Our findings of brandspecific VE are new findings that were made possible by the widespread use of both vaccines in China and worldwide. Second, VEs were effective against pneumonia and severe cases caused by Delta variant compared with another ancestral variant.

This study was subject to some limitations. Because $90 \%$ of people living with HIV or who had tuberculosis had not been vaccinated, our study could not estimate VE in these special populations. Since these two special populations were high-risk groups for infection, their presence in the close-contact subjects may potentially impact the effectiveness of the vaccine. As an observational study, there may have been unmeasured confounding variables that could affect VE estimates. The study was conducted in a limited area in small-scale outbreak, which limited its sample size and therefore the ability to perform subgroup analyses. Also, rigorous non-pharmaceutical interventions may have affected $\mathrm{VE}$ in unknown directions. Finally, the durations from last dose to exposure were all less than 6 months, and most were concentrated in length around 2-3 months; although we found no decline in VE over time, none of the close contacts in our study had been vaccinated more than 6 months prior to becoming a close contact. Therefore, longer duration of effectiveness could not be shown in our study.

In conclusion, completed primary vaccination with 2 doses of the inactivated COVID-19 vaccines was effective against symptomatic COVID-19, COVID-19 pneumonia, and severe COVID-19 caused by the Delta variant of SARS-CoV-2 within 6 months. There were no significant differences in effectiveness of the 2 inactivated COVID-19 vaccines we evaluated.
Although completed primary series with the same brand is recommended, combined primary series appear to be as effective. COVID-19 vaccine coverage of people over 60 years of age and potentially immunocompromised individuals needs to be improved.

doi: $10.46234 / \mathrm{ccdcw} 2022.009$

\# Corresponding authors: Zundong Yin, yinzd@chinacdc.cn; Zhijie An, anzj@chinacdc.cn.

\footnotetext{
${ }^{1}$ Chinese Center for Disease Control and Prevention, Beijing, China; 2 Henan Provincial Disease Control and Prevention, Zhengzhou, Henan, China; ${ }^{3}$ Akesu Prefectural Center for Disease Control and Prevention, Akesu, Xinjiang Uygur Autonomous Region, China; ${ }^{4}$ China Field Epidemiology Training Program, Chinese Center for Disease Control and Prevention, Beijing, China.

\& Joint first authors.
}

Submitted: November 17, 2021; Accepted: January 17, 2022

\section{REFERENCES}

1. COVID-19 Vaccine Technical Working Group. Technical vaccination recommendations for COVID-19 vaccines in China (First Edition). China CDC Wkly 2021;3(21):459 - 61. http://dx.doi.org/10.46234/ ccdcw2021.083.

2. Liu FF, Zheng CJ, Wang LP, Geng MJ, Chen H, Zhou S, et al. Interpretation of the protocol for prevention and control of COVID-19 in China (Edition 8). China CDC Wkly 2021;3(25):527 - 30. http://dx. doi.org/10.46234/ccdcw2021.138.

3. National Health Commission of China. Diagnosis and Treatment Protocol for Novel Coronavirus Pneumonia (Trial Version 8). http:// www.gov.cn/zhengce/zhengceku/2021-04/15/content_5599795.htm. [2022-1-10]. (In Chinese).

4. Al Kaabi N, Zhang Y, Xia S, Yang YK, Al Qahtani MM, Abdulrazzaq N, et al. Effect of 2 inactivated SARS-CoV-2 vaccines on symptomatic COVID-19 infection in adults: a randomized clinical trial. JAMA 2021;326(1):35 - 45. http://dx.doi.org/10.1001/jama.2021.8565.

5. Palacios R, Batista AP, Albuquerque CSN, Patińo EG, Santos JDP, Conde MTRP, et al. Efficacy and safety of a COVID-19 inactivated vaccine in healthcare professionals in Brazil: the PROFISCOV study. https://papers.ssrn.com/sol3/papers.cfm?abstract_id=3822780. [2021-4$11]$

6. Javier SV, Percy SB, Stefan EA, Manuel FN, Miguel MP, Lely S et al. BBIP-CorV vaccine effectiveness for preventing infection and death of health workers, Peru 2021 . https://repositorio.ins.gob.pe/xmlui/bits tream/handle/INS/1318/Efectividad\%20de\%20la.pdf. [2021-12-26] (in Spanish).

7. Wilder-Smith A, Mulholland K. Effectiveness of an inactivated SARSCoV-2 vaccine. N Engl J Med 2021;385(10):946 - 8. http://dx.doi.org/ 10.1056/NEJMe2111165.

8. Ming Kang YY, Li Y, Sun LM, Deng AP. Effectiveness of inactivated COVID-19 vaccines against COVID-19 pneumonia and severe illness caused by the B.1.617.2 (Delta) variant: Evidence from an outbreak in Guangdong, China. Lancet, 2021. preprint. https://papers.ssrn.com/ sol3/papers.cfm?abstract_id $=3895639$.

9. Bernal JL, Andrews N, Gower C, Gallagher E, Simmons R, Thelwall S, et al. Effectiveness of COVID-19 vaccines against the B. 1.617.2 (Delta) variant. N Engl J Med 2021;385(7):585-94. http://dx.doi.org/10. 1056/NEJMoa2108891. 